\title{
Controle do ruído: percepções da equipe e intervenção educativa em Unidade de Terapia Intensiva Neonatal
}

\section{Noise control: team perceptions and educational intervention in a Neonatal Intensive Care Unit}

\section{Control del ruido: Percepciones del Equipo e Intervención Educativa en Unidad de Terapia Intensiva Neonatal}

Fabiana Jorge Bueno Galdino Barsam ${ }^{1}$ Cinthia Lorena Silva Barbosa Teixeira²

Recebido: 02/02/2018 Aprovado: 28/06/2017 Publicado: 01/08/2018
Cláudia Rodrigues de Oliveira ${ }^{3}$ Larissa Cristina de Sousa Lima ${ }^{4}$ Débora de Oliveira Ferreira ${ }^{5}$ Maria Sueli de Souza Silva ${ }^{6}$ Fernanda Carolina Camargo ${ }^{7}$

Estudo híbrido, composto de fase transversal-quantitativa, realizado de março a maio de 2017, através de levantamento e fase descritiva-narrativa de abordagem qualitativa, que teve como objetivo identificar a percepção do ruído de uma Unidade de Terapia Intensiva Neonatal de hospital público de ensino e descrever ações de Educação Permanente em Saúde (EPS) empreendidas para o controle do ruído. Foram identificados como principais efeitos do ruído na equipe: irritabilidade (83,3\%), dores de cabeça $(75 \%)$ e perda de atenção $(68,3 \%)$. Principais fontes: alarmes de monitores e incubadoras (95\%) e conversas na unidade (85\%). A formação de Grupo Condutor com expertise, reuniões em diferentes turnos e a flexibilização da participação foram iniciativas viabilizadoras para a implementação da EPS. Foi elaborado um menu de sugestões apoiadoras ao desenvolvimento da "cultura do silêncio" no setor. Mediante o desafio mundial quanto ao controle de ruídos nestas unidades, inferese que a descrição do processo e das iniciativas favorece sua replicação em cenários semelhantes.

Descritores: Monitoramento do ruído; Unidades de terapia intensiva neonatal; Hospitais de ensino.

This is a hybrid study, with a quantitative and cross-sectional stage, conducted from March to May 2017 through a survey, and a descriptive-narrative qualitative stage, aimed at identifying noise perception in a Neonate Intensive Care Unit at a public teaching hospital, and describing actions of Permanent Health Education (PHE) used for noise control. The main effects the noise was found to have on the team were: irritability (83.3\%), headaches (75\%) and loss of attention (68.3\%). Main sources of noise: monitor and incubator alarms (95\%) and conversation in the unit $(85 \%)$. The formation of a Guiding Group with expertise on the field, meetings in different turns and flexibilization of participation were the initiatives that enabled the implementation of the PHE. A list of suggestions to support the development of the "culture of silence" in the sector was elaborated. Considering the worldwide challenge regarding noise control in these units, it can be inferred that the description of the process and initiatives will enable its replication in similar settings. Setembro

Descriptors: Noise monitoring; Intensive care units neonatal; Hospitals teaching.

Estudio híbrido, compuesto de fase transversal-cuantitativa, realizado de marzo a mayo de 2017, a través de levantamiento y fase descriptiva-narrativa de abordaje cuantitativo, que tuvo como objetivo identificar la percepción del ruido de una Unidad de Terapia Intensiva Neonatal de hospital público de enseñanza y describir acciones de Educación Permanente en Salud (EPS) emprendidas para el control del ruido. Fueron identificados como principales efectos del ruido en el equipo: irritabilidad (83,3\%), dolor de cabeza (75\%) y pérdida de atención (68,3\%). Principales fuentes: alarmas de monitores e incubadoras $(95 \%)$ y conversaciones en la unidad (85\%). La formación de Grupo Conductor con experiencia, reuniones en diferentes turnos y la flexibilización de la participación fueron iniciativas viabilizadoras para la implementación de la EPS. Fue elaborado un menú de sugerencias apoyadoras para el desarrollo de la "cultura del silencio" en el sector. Mediante el desafío mundial en cuanto al control de ruidos en estas unidades, se infiere que la descripción del proceso y de las iniciativas favorece su replicación en escenarios semejantes.

Descriptores: Monitoreo del ruido; Unidades de cuidado intensivo neonatal, Hospitales de enseñanza.

1. Médica Neonatologista e Pediátrica. Doutora em Ciências da Saúde. Professora Adjunta do curso de Graduação em Medicina. Departamento Materno Infantil e de Pediátria da Universidade Federal do Triângulo Mineiro (UFTM). Uberaba, MG, Brasil. ORCID: 0000-0002-8143-4083 E-mail: faturka2002@hotmail.com

2. Enfermeira. Especialista em Enfermagem em Unidade de Terapia Intensiva Neonatal e Pediátrica. MBA em Gestão Hospitalar. Enfermeira do Hospital de Clínicas (HC) da UFTM. Uberaba, MG, Brasil. ORCID: 0000-0001-7864-5528 E-mail: cinthiabarbosa.enf@gmail.com

3. Enfermeira. Especialista em Enfermagem Neonatal e Pediátrica. Enfermeira do HC-UFTM. Uberaba, MG, Brasil. ORCID: 0000-0002-1992-4735 E-mail: claudinhaenfermagem6@hotmail.com

4. Fonoaudióloga. Especialista em Voz. Fonoaudióloga do HC-UFTM. Uberaba, MG, Brasil. ORCID: 0000-0003-1449-8789 E-mail: larissalima_fonoaudiologia@hotmail.com

5. Enfermeira. Especialista em Enfermagem em Neonatologia. Mestranda em Atenção à Saúde pela UFTM. Enfermeira do HC-UFTM. Uberaba, MG, Brasil. ORCID: 0000-0003-3685-0800 E-mail: deboradeoliveiraferreira@hotmail.com

6. Grupo de Humanização e Acolhimento Familiar do HC-UFTM. Uberaba, MG - Brasil. ORCID: 0000-0003-0386-6822 E-mail: sueli.enf@outlook.com

7. Enfermeira. Doutora em Atenção à Saúde. Epidemiologista Clínica do Setor de Pesquisa e Inovação Tecnológica da Gerencia de Ensino e Pesquisa do HC-UFTM.

Uberaba, MG Brasil. ORCID: 0000-0002-1048-960X E-mail: fernandaccamargo@yahoo.com.br 


\section{INTRODUÇÃO}

A Unidade de Terapia Intensiva Neonatal (UTIPN) é um ambiente hospitalar de elevada complexidade, onde se cuida de recém-nascidos em estado grave. Uma das premissas atuais do cuidado em neonatologia é a necessidade de se minimizar o manuseio e empreender controles ambientais para o adequado restabelecimento dos recém-nascidos, haja vista que os prematuros correm maior risco de desenvolverem distúrbios cognitivos, motores e comportamentais em comparação aos recém-nascidos a termo ${ }^{1,2}$.

A equipe multiprofissional da UTIPN tem de ser competente em manejar tecnologias (monitores, incubadoras, bombas de infusão, entre outros) e trabalha em um ambiente em que está condicionado ao estresse, devido à instabilidade dos neonatos, e sua baixa tolerância a erros mediante procedimentos de alto risco e barulho excessivo ${ }^{3,4}$. Nesta perspectiva, o controle do ruído é uma ação que ganha destaque entre as equipes multiprofissionais atuantes na UTIPN $^{1,2}$.

Observa-se que o ruído nos cenários de UTIPN é mais alto do que na maioria dos ambientes. Esses sinais auditivos têm sido concebidos como perturbadores e ocorrem em intervalos irregulares. Frequentemente, causam problemas aos recém-nascidos, seus familiares e aos trabalhadores do setor ${ }^{1,4}$ especialmente quando o controle inadequado do ruído nesses ambientes, durante a prestação do cuidado, pode acarretar danos aos neonatos, sendo eles de natureza física, social e/ou psicológica. Uma revisão sistemática recente demonstrou a importância de estratégias para monitoramento do ruído no ambiente da UTIPN ${ }^{1}$.

Por conseguinte, a existência desse controle em ambiente de UTIPN tem sido ação de interesse em hospitais de ensino em diferentes localidades mundiais 5,6 . Estudos têm apontado valores aferidos acima dos limites de segurança recomendados, entre 35$45 \mathrm{~dB}$, como exemplificado por uma experiência norte-americana para controle do ruído em UTI-Neo, cujas medições prévias à intervenção apresentaram valores médios de $57.0 \mathrm{~dB}$ (desvio padrão $\pm 0,84$ ) ${ }^{5}$. Em um hospital de ensino no México, as aferições revelaram uma média de $61.8 \mathrm{~dB}$ (desvio padrão $\pm 4,4)^{7}$. Em um hospital canadense, um sistema de auditoria identificou limites entre 45 ou $55 \mathrm{~dB}$, no setor em questão do hospital ${ }^{6}$.

Ao se tratar de Hospitais Públicos de Ensino brasileiros, os desafios para o controle do ruído são ainda maiores. Primeiro, por serem espaços de elevada circulação de pessoas. Tradicionalmente, convivem neste espaço, trabalhadores, docentes, pesquisadores e estudantes com diferentes níveis de formação ${ }^{8}$.

Ainda, a cultura organizacional desses hospitais apresenta elementos relacionados a sua rígida estrutura e à centralização de poder; resultando em dificuldades para o desenvolvimento do trabalho em equipe e o engajamento participativo dos trabalhadores para mudanças 9 . Contudo, essas fragilidades são resultantes de influências, no âmbito da administração do serviço hospitalar, de fatores relativos à macroestrutura do próprio sistema de saúde, e de limitações dos trabalhadores quanto a suas qualificações técnicas para o exercício ${ }^{8,9}$.

Como consequência, ocorrem: a ausência de planejamento global da unidade de internação, problemas na coordenação dos processos assistenciais e administrativos, falhas de comunicação e inexistência de instrumentos de gestão participativa 8,10 .

A superação dessas práticas tende a influenciar de forma positiva a qualidade do cuidado e a cultura de segurança do paciente nas instituições hospitalares como um todo, principalmente ao se valorizar a promoção das relações interpessoais, a motivação e a satisfação dos trabalhadores ${ }^{8,10}$. Esses aspectos ganham relevância à medida em que o tema da qualidade do cuidado nos hospitais assume um papel cada vez maior na agenda dos gestores, dos profissionais de saúde e dos usuários, principalmente frente às discussões que envolvem a segurança do paciente 9 .

Em busca de modos propositivos para a superação de demandas clínicas e gerenciais das unidades de internação hospitalares, como as UTIPN, o estabelecimento de espaços 
para Educação Permanente em Saúde (EPS) entre as equipes multiprofissionais tem sido empreendido como estratégia para sobrepor tais desafios.

Sumariamente, a EPS é tida como a produção de conhecimento no cotidiano das instituições de saúde, a partir da realidade vivenciada pelos atores envolvidos, sendo a problematização da prática assistencial e as experiências das equipes envolvidas, tidas como base para interrogação, mudança e elaboração de proposição de intervenções participativas na transformação das realidades ${ }^{11}$.

Este estudo tem como objetivo identificar a percepção do ruído de uma Unidade de Terapia Intensiva Neonatal de um hospital público de ensino, e descrever ações de Educação Permanente em Saúde empreendidas para o controle do ruído.

\section{MÉTODO}

Trata-se de um estudo com delineamento híbrido ${ }^{12}$ e exploratório, composto de duas fases: a primeira caracterizada por um estudo transversal-quantitativo, tendo como base um levantamento organizado por meio de um questionário estruturado autoaplicável elaborado pelos autores; e a segunda por uma pesquisa descritiva e abordagem qualitativa, sobre o empreendimento da EPS no setor.

O cenário de estudo foi uma UTIPN composta por 20 leitos de hospital geral, público e de ensino, de grande porte (332 leitos) - referência macrorregional para a alta complexidade assistencial do polo Triângulo Sul de Minas Gerais, Brasil.

O desenvolvimento do projeto foi motivado pelo interesse em apoiar os objetivos estratégicos do hospital, o qual tem como missão garantir a qualidade assistencial e a segurança do paciente frente a articulação entre ensino-pesquisa-extensão e assistência. A pesquisa foi desenvolvida entre março e maio de 2017.

Foram distribuídos 120 questionários (número total de profissionais que atuam no setor, independente da categoria profissional), abrangendo a equipe multidisciplinar: médicos, residentes médicos, enfermeiros (incluindo residentes de enfermagem), técnicos de enfermagem, fisioterapeutas, psicóloga, fonoaudióloga, equipe de limpeza e apoio, escriturário. 0 intuito foi a composição de uma amostra nãoprobabilística, por meio da maximização de participações.

Os participantes foram orientados sobre a devolução do instrumento em até 48 horas após recebimento. As questões estruturadas abordavam: caracterização sociodemográfica dos participantes; como eles caracterizavam o nível do ruído; sinais e sintomas que consideravam serem causados pela exposição ao ruído exagerado; e fontes que consideravam causar ruído excessivo na UTIPN. Foi organizado um banco de dados e em seguida empreendeu-se análises por estatística descritiva, conforme frequências absolutas e relativas, utilizado para ambos $o$ software Microsoft Excel@.

Quanto à segunda fase, a técnica descritiva incluiu a análise de registros documentais (atas de reuniões) realizados para a EPS, considerando a identificação dos temas debatidos, as estratégias empreendidas e o engajamento da equipe multiprofissional no processo. A análise partiu da identificação do processo, por meio do estudo das ações do grupo (equipe multiprofissional), realizando um exame dos registros e buscando compreender o fenômeno em termos das situações, eventos e os processos que os conectam ${ }^{13}$.

Sendo a EPS o próprio arcabouço orientador teórico-crítico desta fase, pode-se considera-la como uma prática educativa, ativa e problematizadora para a formação em serviço, ancorada no trabalho, no conhecimento prévio dos trabalhadores, na aprendizagem significativa e na transformação da prática. Em muito, o referencial teórico da EPS permite ao trabalhador de saúde intervir em seu contexto de forma crítica, coletiva e integradora ${ }^{11}$.

Os resultados correspondentes foram apresentados de forma sumarizada e em quadros. Quanto ao aspecto ético, foi respeitada a Resolução CNS 466/2012, pesquisa aprovada pelo Comitê de Ética em Pesquisa da Universidade Federal do 
Triângulo Mineiro, sob o parecer $\mathrm{n}^{\mathrm{o}}$ 1.1618.872.

\section{RESULTADOS}

$\mathrm{Na}$ primeira fase do estudo, houve a devolutiva de 60 instrumentos, na seguinte distribuição: técnicos de enfermagem $(n=22)$, médicos $\quad(n=14)$, enfermeiros $(n=13)$, fisioterapeutas $(n=9)$, escriturários $(n=2)$ e fonoaudióloga $(n=1)$, correspondendo a $50 \%$ o retorno dos questionários preenchidos.

A maior parte dos respondentes $(63,3 \%)$ tem atuado na UTIPN de um a cinco anos. Consideraram que o ruído na unidade se apresenta como alto (55\%) e muito alto $(33,3 \%)$, sendo que apenas sete dentre eles $(11,7 \%)$ o percebem como normal.

Quanto aos sinais e sintomas que apresentavam frente à exposição ao ruído exagerado foram apontados com maior frequência: irritabilidade $(83,3 \%)$, dores de cabeça (75\%), perda de atenção $(68,3 \%)$ e dificuldades de relaxamento psíquico $(66,7 \%)$ (Tabela 1).

Tabela 1. Sinais e sintomas recorrentes entre os trabalhadores da Unidade de Terapia Intensiva Neonatal frente a exposição ao ruído inadequado. Uberaba, MG, 2017.

\begin{tabular}{lll}
\hline Sinais e Sintomas & $\mathbf{n}^{\mathbf{a}}$ & $\mathbf{\%}$ \\
\hline Irritabilidade & 50 & 83,3 \\
Dores de cabeça & 45 & 75,0 \\
Perda de Atenção & 41 & 68,3 \\
Dificuldade de relaxamento psíquico & 40 & 66,7 \\
Alterações na qualidade do sono & 31 & 51,7 \\
Problemas na comunicação com colegas & 31 & 51,7 \\
Alucinações auditivas & 28 & 46,7 \\
Fadiga & 26 & 43,3 \\
Dores musculares tensionais & 20 & 33,3 \\
Perda auditiva & 19 & 31,7 \\
Taquicardia & 15 & 25,0 \\
Perda do senso crítico e de julgamento & 15 & 25,0 \\
Depressão/ ansiedade & 14 & 23,3 \\
Maior liberação de ácido estomacal /gastrite & 11 & 18,3 \\
Hipertensão arterial & 9 & 15,0 \\
\hline aCada respondente poderia assinalar mais de uma opção & &
\end{tabular}

Sobre as fontes geradoras de ruídos excessivos, foram percebidas como principais fontes: alarmes de monitores e incubadoras (95\%, respectivos), conversas na unidade
(85\%), o mover de cadeiras e mesas (83,3\%), as bombas de infusão (80\%) e o fechamento de portinholas $(76,7 \%)$ (Tabela 2$)$. 
Tabela 2. Fontes geradoras de ruídos excessivos conforme percepção dos trabalhadores da Unidade de Terapia Intensiva Neonatal. Uberaba/MG, 2017.

\begin{tabular}{lcc}
\hline Fontes & $\mathbf{n}^{\mathbf{a}}$ & $\mathbf{\%}$ \\
\hline Alarmes dos monitores & 57 & 95,0 \\
Alarme da incubadora (sobreaquecimento) & 57 & 95,0 \\
Conversas na unidade & 51 & 85,0 \\
Mover cadeiras e mesas & 50 & 83,3 \\
Bombas de infusão & 48 & 80,0 \\
Fechamento das portinholas & 46 & 76,7 \\
Abrir e fechar as lixeiras & 43 & 71,7 \\
Colocar materiais em cima da incubadora & 42 & 70,0 \\
Toque do telefone & 39 & 65,0 \\
Ventilador & 38 & 63,3 \\
Abrir e fechar de portas & 37 & 61,7 \\
Bombas de seringa & 37 & 61,7 \\
Vácuo & 35 & 58,3 \\
Uso do celular dentro da unidade & 34 & 56,7 \\
Fechar gavetas & 34 & 56,7 \\
Sapato ruidoso & 33 & 55,0 \\
Choro dos pacientes & 31 & 51,7 \\
CPAP & 30 & 50,0 \\
Água no circuito de ventilação mecânica & 30 & 50,0 \\
Ajuste da temperatura da incubadora & 29 & 48,3 \\
Aparelho de raio X & 28 & 46,7 \\
Ar condicionado & 27 & 45,0 \\
Fechar painel de acesso da incubadora & 26 & 43,3 \\
Suporte de papel toalha & 25 & 41,7 \\
Torneira aberta & 21 & 35,0 \\
Impressora & 9 & 15,0 \\
\hline aCada respondente poderia assinalar mais de uma opcão & &
\end{tabular}

Quanto à segunda fase da pesquisa, conforme a análise documental, para se alcançar o desenvolvimento da EPS foi observada a organização de um pequeno grupo composto por trabalhadores do próprio setor que tinham expertise nesse tipo de implantação.

Os integrantes deste Grupo Condutor (GC) do projeto foram: a Responsável Técnica (RT) de Enfermagem e o RT Médico, um Representante da Comissão de Controle de Infecção Hospitalar (CCIH), um Representante da Unidade de Gerenciamento de Resíduos, dois Representantes da Engenharia Clínica, o Responsável Técnico pelo Setor de Radiologia, a Responsável Técnica pelo Setor de Nutrição Enteral, o preceptor dos Programas de Residência de Enfermagem
Multiprofissional do Hospital e o Staff do Programa de Residência Médica do Setor.

0 engajamento para EPS exigiu a realização de reuniões nos diferentes turnos, pelos integrantes do GC. 0 convite aos trabalhadores da UTIPN para a participação nas reuniões foi feito por meio da disponibilização de informações no quadro de avisos do setor, incluindo datas, horários e objetivos de cada encontro.

Foram disponibilizados previamente os temas a serem discutidos, e a duração estimada de cada encontro foi de 45 minutos - garantindo melhor controle da rotina assistencial. A seguir descreve-se a participação dos trabalhadores nas reuniões realizadas nos meses de março e maio de 2017, para problematização e discussão temática (Quadro 1). 
Quadro 1. Caracterização das reuniões para educação permanente em saúde sobre o controle do ruído na Unidade de Terapia Intensiva Neonatal. Uberaba/MG, 2017.

\begin{tabular}{|c|c|c|c|}
\hline Temas & Objetivos & $\begin{array}{c}\text { Técnicas } \\
\text { Empreendidas }\end{array}$ & $\begin{array}{c}\text { Categorias } \\
\text { Participantes (n) }\end{array}$ \\
\hline $\begin{array}{l}\text { Como } \\
\text { está o } \\
\text { ruído na } \\
\text { UTIPN? }\end{array}$ & $\begin{array}{c}\text { Discutir, de forma } \\
\text { coletiva, os resultados } \\
\text { do levantamento por } \\
\text { questionários }\end{array}$ & $\begin{array}{c}\text { Exposição } \\
\text { dialogada orientada } \\
\text { por apresentação } \\
\text { de slides }\end{array}$ & $\begin{array}{c}\text { Enfermeiros (6) } \\
\text { Escriturário (1) } \\
\text { Fisioterapeuta (1) } \\
\text { Médicos (14) } \\
\text { Profissional da limpeza (1) } \\
\text { Técnicos de enfermagem } \\
\text { (38) }\end{array}$ \\
\hline $\begin{array}{l}\text { Como } \\
\text { controlar } \\
\text { o ruído } \\
\text { na } \\
\text { UTIPN? }\end{array}$ & $\begin{array}{l}\text { Identificar, de forma } \\
\text { coletiva, ações que } \\
\text { estão sob a } \\
\text { governabilidade da } \\
\text { equipe para o melhor } \\
\text { gerenciamento do } \\
\text { ruído } \\
\end{array}$ & $\begin{array}{c}\text { Exposição } \\
\text { dialogada orientada } \\
\text { por apresentação } \\
\text { de slides e caixa de } \\
\text { sugestões }\end{array}$ & $\begin{array}{c}\text { Enfermeiros (6) } \\
\text { Escriturário (1) } \\
\text { Fisioterapeuta (1) } \\
\text { Médicos (14) } \\
\text { Profissional da limpeza (1) } \\
\text { Técnicos de enfermagem } \\
\text { (43) }\end{array}$ \\
\hline
\end{tabular}

Para o empreendimento das reuniões, o GC teve como preocupação - valorizar o trabalho em equipe, seguindo a perspectiva do referencial teórico da EPS. A comunicação utilizada pretendeu ser clara, buscando reduzir mal-entendidos; a aprendizagem buscou ultrapassar a transferência de saber de uma pessoa a outra, valorizando-se o aprender com o outro e não do outro.

Com isso, buscou-se construir espaços de cooperação, favorecendo a articulação entre os integrantes da equipe, de seus saberes, e de seus fazeres. E, ainda, o GC buscou empreender uma rede de acolhimento - para que o clima durante as reuniões não fosse tenso ou de disputas.

É importante destacar que, após a segunda reunião, o GC responsabilizou-se por ampliar a sensibilização de todos os trabalhadores quanto às temáticas discutidas. Para tanto, seus membros se responsabilizaram por captar, em momentos externos às reuniões, como os demais trabalhadores do setor apresentariam sugestões para a superação do problema.

As sugestões foram organizadas em lista, e o material serviu de embasamento para a definição de ações no setor conforme o planejamento previsto na unidade. Foram elencadas para a implementação aquelas que apresentavam maior governabilidade da equipe, em especial quanto a recursos críticos ao seu desenvolvimento, como: financeiros, organizacionais e de conhecimento. 0 menu de sugestões foi registrado em ata de EPS no setor, e reuniões subsequentes discutiram a elaboração de um cronograma e dos responsáveis pela implantação das ações. Descrevem-se as ações:

a) Sensibilizar toda a equipe, incluindo médicos e plantonistas de final de semana sobre a necessidade do controle de ruído na unidade;

b) Sensibilizar todas as equipes que promovem cuidados na UTIPN (como equipe do lactário, da copa, dos especialistas em interconsulta) e familiares dos neonatos sobre o controle de ruídos;

c) Orientar a equipe sobre evitar o uso de sapatos ruidosos no setor;

d) Orientar e sensibilizar a equipe para mudanças de comportamento como: evitar arrastar cadeiras, fechar portas dos armários e gavetas com cautela, abrir e fechar portinholas cuidadosamente, não colocar objetos sobre a incubadora, evitar conversas paralelas, entre outros;

e) Orientar a equipe médica a atender prontamente ao telefone, principalmente durante os horários estabelecidos para notícias telefônicas aos pais/familiares;

f) Exigir que celulares ou outros dispositivos eletrônicos para comunicação estejam neste 
ambiente somente no modo silencioso, inclusive para os familiares;

g) Solicitar autorização para diminuição do volume dos alarmes dos equipamentos, e sua padronização, na UTI-Neo;

h) Transferir a impressora do centro da unidade para a sala de guarda de incubadoras;

i) Engajar as equipes médica e de enfermagem na implantação de medidas para abordagem do choro incontrolável do bebê, adotando o consolo por meio de medidas simples (acalento, mudança de posicionamento, sucção não nutritiva, dentre outras).

\section{DISCUSSÃO}

A temática controle de ruídos em UTIPN tem se destacado na literatura contemporânea ${ }^{13}$. Utilizando o método Delphi para identificar indicadores de qualidade sensíveis à atuação de enfermeiros nessas unidades, pesquisadores chineses apresentaram o controle do ruído como algo de elevada importância ${ }^{14}$.

A submissão ao ruído excessivo nesses setores tem apresentado efeitos deletérios à saúde dos trabalhadores, podendo acarretar até mesmo a síndrome de burnout, por facilitar a aparição de sintomas como estresse, irritabilidade, fadiga e instabilidade emocional na equipe que atua no setor ${ }^{15}$.

Resultados convergentes à presente pesquisa foram identificados em um hospital público universitário de Governador Valadares, MG, onde 235 integrantes da equipe multiprofissional identificaram como efeitos deletérios do ruído inadequado sentirem desconforto quando submetidos a sons fortes $(75,7 \%)$, mal-estar ao final do expediente (35,3\%), e dificuldades em ouvir o que as pessoas dizem $(29,8 \%)^{16}$. Em um hospital universitário do estado do Rio de Janeiro, os equipamentos de uma forma geral apresentaram medidas sonoras acima do volume de segurança em todos os diferentes turnos, quando avaliados em uma rotina habitual da equipe multiprofissional de uma UTIPN17.

Quanto às propostas de intervenção para a mudança frente ao controle do ruído no âmbito da UTIPN, aponta-se a necessidade de que esse ambiente seja regido pelo silêncio ${ }^{18,19}$, sendo o emprego de momentos de total quietude nesses setores crucial ao desenvolvimento dos neonatos assistidos. Doravante, os profissionais reconhecerem quais eram os cuidados essenciais para o gerenciamento do ruído nestes cenários. 0 desafio à sua implantação permeia, sobremaneira, a cultura organizacional que perfaz a UTIPN ${ }^{19-21 . ~}$

Essa cultura compreende as experiências que os trabalhadores vivenciam nos ambientes hospitalares e impacta a segurança do sistema analisado principalmente quando as maiores fragilidades e fortalezas do mesmo são reconhecidas. 0 desenvolvimento dos aspectos relacionados à comunicação e à cooperação dentro dos serviços de saúde é tido como essencial para que o sistema possa ser guiado a oportunidades de melhoria da atenção, e por conseguinte, da segurança às pessoas assistidas e da satisfação com 0 trabalho da equipe de UTIPN 21 .

Ao se discutir a sustentabilidade da cultura do silêncio nos ambientes de UTIPN durante situações não emergenciais, podem ser observadas como essenciais a implementação e a difusão de estratégias, tais como aquelas que favorecem 0 reconhecimento dos efeitos deletérios do ruído nos neonatos e na equipe.

É preciso que a modificação do manejo do ruído seja ação expandida a toda equipe multidisciplinar - pois quando restrita à equipe de enfermagem, a sustentabilidade da mudança favorável à cultura do silêncio se torna reduzida. Em consequência, a adesão de todos os trabalhadores atuantes no setor e o senso de cooperação devem integrar as iniciativas para a implantação dessa cultura 20,21 .

Nesta perspectiva, para mudanças e engajamento da equipe, o processo de trabalho em saúde deve ser cada vez mais ancorado em saberes compartilhados, de modo que os trabalhadores consigam incorporar mudanças na estrutura do trabalho. Com isso, a utilização da EPS como referencial para o alcance dessas mudanças parte de uma problematização da realidade local que é o 
ponto gerador de construções coletivas para a mudança, sendo também capaz de evitar o delineamento de ações descontextualizadas e levar a iniciativas que tenham um respaldo prático em sua execução 22,23 .

Sobretudo, a EPS tem sido estabelecida e orientada por pensamento livre, crítico e reflexivo, utilizado para transformar a prática vivenciada e fundamentar um compromisso pessoal e profissional frente a essa transformação ${ }^{22,23}$.

A EPS tornou-se um importante instrumento de mudança quando direcionada para a área da saúde, além de aproximar os diversos sujeitos integrantes do cuidado para auxiliarem em sua melhoria. Como desafio às instituições hospitalares para a implantação da EPS apresenta-se a falta de interesse dos profissionais, a falta de tempo e o pouco incentivo dos gestores das instituições ${ }^{23}$.

Conforme identificado na presente pesquisa, as estratégias empreendidas, como a formação de um GC, o estabelecimento de reuniões nos diferentes turnos, com aviso prévio e flexibilização da participação, levaram a iniciativas que viabilizaram a realização da EPS, a discussão da prática de trabalho e, por conseguinte, a construção de orientações para a mudança da realidade.

A limitação do estudo apresenta-se quanto à generalização dos resultados, frente ao retorno dos respondentes da primeira fase e o menu de sugestões ter sido elaborado contextualizado à realidade específica de uma UTIPN. Entretanto, a partir das iniciativas descritas, desde o levantamento dos efeitos do ruído na equipe e no ambiente da UTI-Neo, até a implantação da EPS enquanto estratégia para orientar a mudança e as sugestões registradas, infere-se que tais ações poderiam ser empreendidas em cenários semelhantes ao hospital de ensino analisado.

\section{CONCLUSÃO}

Mediante o desafio mundial relacionado ao controle de ruídos em cenários de UTIPN, ao se considerar os efeitos deletérios que os ruídos excessivos acarretam aos trabalhadores e aos neonatos assistidos, a presente pesquisa apresenta colaboração para análise e proposições para seu enfrentamento. De forma geral, os resultados apresentam-se como inovadores quando se discute a utilização do referencial da EPS para operacionalização da mudança no setor, no sentido do alcance de uma cultura do silêncio.

Apesar do baixo retorno dos respondentes na primeira fase da pesquisa, limitando a generalização dos achados, os resultados apresentados convergiram na mesma direção de pesquisas realizadas em cenários semelhantes.

Ainda, subsidiaram a problematização que levou à gênese das construções compartilhadas e do menu de iniciativas, propostas da equipe multiprofissional consoantes ao referencial da EPS. Ademais, a descrição do processo de condução das iniciativas empreendidas favorece a replicação do método em cenários semelhantes para que efeitos similares possam ser alcançados.

Contudo, são necessárias pesquisas futuras que visem identificar a real implantação da cultura do silêncio no setor mediante a implantação do menu de sugestões. Sugere-se, para tanto, que seja realizado um diagnóstico quanto aos níveis de ruído, mapeados conforme turnos e fontes, prévia e posteriormente à implementação do menu de sugestões.

Outras avaliações importantes diriam respeito à percepção de ruídos e de seus efeitos deletérios entre os trabalhadores, o tempo de internação dos neonatos na UTIPN e a satisfação das equipes e familiares frente as modificações implementadas.

\section{REFERÊNCIAS}

1. Almadhoob A, Ohlsson A. Sound reduction management in the neonatal intensive care unit for preterm or very low birth weight infants. Cochrane Database Syst Rev. [Internet]. 2015 [citado em: 18 set 2017]; 1:CD010333. Disponível em: http://cochranelibrarywiley.com/doi/10.1002/14651858.CD01033 3.pub2/abstract;jsessionid=391AA94C6DA5 92798F06BECD640824CA.f04t04

2. Montanholi LL, Merighi MAB, Jesus MCP. Atuação da enfermeira na unidade de terapia intensiva neonatal: entre o ideal, o real e o 
possível. Rev Latinoam Enferm. [Internet]. 2011 [citado em: 18 set 2017]; 19(2):08 telas. Disponível em: http://www.scielo.br/pdf/rlae/v19n2/pt_11 3. Vieira FPC, Garcia PC, Fugulin FMT. Tempo de assistência de enfermagem e indicadores de qualidade em Unidade de Terapia Intensiva pediátrica e neonatal. Acta Paul Enferm. [Internet]. 2016 [citado em: 18 set 2017]; 29(5):558-64.

http://dx.doi.org/10.1590/19820194201600077

4. Daniel VP, Silva JSLG. A Enfermagem e sua colaboração na Unidade de Terapia Intensiva Neonatal. Rev Pró-UniverSUS [Internet]. 2017 [citado em 19 set 2017]; 08(1):3-7. Disponível em:

http://editorauss.uss.br/index.php/RPU/arti cle/view/687/687

5. Chawla S, Barach P, Dwaihy M, Kamat D, Shankaran S, Panaitescu B, et al. A targeted noise reduction observational study for reducing noise in a neonatal intensive unit. J Perinatol. [Internet]. 2017 [citado em: 17 set 2017]; 37(9):1060-4. DOI: 10.1038/jp.2017.93

6. Wang D, Aubertin C, Barrowman N, Moreau K, Dunn S, Harrold J. Reduction of noise in the neonatal intensive care unit using soundactivated noise meters. Arch Dis Child Fetal Neonatal Ed. [Internet]. 2014 [citado em: 20 set 2017]; 99(06):F515-6. Disponível em: http://fn.bmj.com/content/fetalneonatal/99 /6/F515.full.pdf DOI: 10.1136/archdischild2014-306490

7. Nieto-Sanjuanero A, Quero-Jiménez J, Cantú-Moreno D, Rodríguez-Balderrama I, Montes-Tapia F, Rubio-Pérez N, et al. Evaluation of strategies aimed at reducing the level of noise in different areas of neonatal care in a tertiary hospital. Gac Med Mex. [Internet]. 2015 [citado em: 18 set 2017]; 151:687-94. Disponível em: http://www.anmm.org.mx/GMM/2015/n6_e nglish/2331AX156_151_2015_UK6_687-

694.pdf

8. Brehmer LCF, Ramos FRS. Teaching-service integration: implications and roles in experiences of Undergraduate Courses in Nursing. Rev Esc Enferm USP. [Internet]. 2014 [citado em: 18 set 2017]; 48(1):118-24. DOI:
http://dx.doi.org/10.1590/S0080-

623420140000100015

9. Rocha FL, Marziale MHP, Carvalho MC, Cardeal SF, Campos MCT. The organizational culture of a Brazilian public hospital. Rev Esc Enferm USP. [Internet]. 2014 [citado em: 18 set 2017]; 48(2):308-14. DOI: http://dx.doi.org/10.1590/S0080-

6234201400002000016

10. Lorenzetti J, Gelbcke FL, Vandresen L. Tecnologia para gestão de unidades de internação hospitalares. Texto \& Contexto Enferm. [Internet]. 2016 [citado em: 20 set 2017]; 25(2):e1770015. DOI: http://dx.doi.org/10.1590/0104-

07072016001770015

11. Gigante RL, Campos GWS. Política de formação e educação permanente em saúde no brasil: bases legais e referências teóricas. Trab Educ Saúde. [Internet]. 2016 [citado em: 18 set 2017]; 14(3):747-63. DOI: http://dx.doi.org/10.1590/1981-7746sip00124

12. Abedellah FG, Levine E. Better patient care through nursing research. New York: MacMillan; 1965.

13. Martind HHTS. Metodologia qualitativa de pesquisa. Educ Pesqui. [Internet]. 2004 [citado em: 17 set 2017]; 30(2):289-300. DOI: http://dx.doi.org/10.1590/S1517-

97022004000200007

14. Chen L, Huang LH, Xing MY, Feng ZX, Shao LW, Zhang MY et al. Using the Delphi Method to develop nursing-sensitive quality indicators for the NICU. J Clin Nurs.

[Internet]. 2017 [citado em: 18 set 2017]; 26(3-4):502-13. Disponível em: https://onlinelibrary.wiley.com/doi/epdf/10 .1111/jocn.13474 DOI: 10.1111/jocn.13474

15. Galindo APG, Caicedo YC, Pereira AV. Nível continuo equivalente de ruído em la unidad de cuidado intensivo neonatal associado al síndrome de burnout. Enferm Intensiva [Internet]. 2015 [citado em: 18 set 2017]; 26(3):92-100.

DOI: http://dx.doi.org/10.1016/j.enfi.2015.03.002 16. Andrade KP, Oliveira LLA, Souza RP, Matos IM. Medida do nível de ruído hospitalar e seus efeitos em funcionários a partir do relato de queixas. Rev CEFAC [Internet]. 2016 [citado em 20 set 2017]; 18(6):1379-88. DOI: 
http://dx.doi.org/10.1590/1982-

0216201618619815

17. Jordão KR, Pinto LAP, Machado LR, Costa LBVL, Trajno ETL. Possible stressors in a neonatal intensive care unit at a university hospital. Rev Bras Ter Intensiva [Internet]. 2016 [citado em: 19 set 2017]; 28(3):310-14. Disponível em: http://www.scielo.br/pdf/rbti/v28n3/en_01 03-507X-rbti-20160041.pdf DOI: http://dx.doi.org/10.5935/0103507X.20160041

18. Laubach V, Wilhelm P, Carter K. Shhh...I'm growing: noise in the NICU. Nurs Clin North Am. [Internet]. 2014 [citado em: 19 set 2017]; 49(3):329-44. Disponível em: https://ac.elscdn.com/S0029646514000371/1-s2.0S0029646514000371-

main.pdf?_tid=c812fcf8-d88b-43ae-aaa206fb2dcf922a\&acdnat $=1526303054$ f71 bedc 8acaab4d7499642e8702fe801 DOI: 10.1016/j.cnur.2014.05.007

19. Mahmoodi N, Arbabisarjou A, Rezaeipoor M, Mofrad ZP. Nurses'Awareness of Preterm Neonates'sleep in the NICU. Glob J Health Sci. [Internet]. 2016 [citado em 18 set 2017]; 8(6):226-33. Disponível em: http://www.ccsenet.org/journal/index.php/ gjhs/article/view/54945/29276

20. Santiago THRS, Turrini RNT. Cultura e clima organizacional para segurança do paciente em unidades de terapia intensiva. Rev Esc Enferm USP. [Internet]. 2015 [citado em 17 set 2017]; 49 (spe):123-30. DOI: http://dx.doi.org/10.1590/S0080-

623420150000700018

21. Swath S, Ramesh A, Nagapoornima M, Fernandes LM, Jisina C, Suman Rao PN, et al. Sustaining a "culture of silence" in the neonatal intensive care unit duringa nonemergency situations: a grounded theory on ensuring adherence to behavioral modification to reduce noise levels. Int J Qual Stud Health Well-being [Internet]. 2014 [citado em 18 set 2017]; 9:22523. DOI: http://dx.doi.org/10.3402/qhw.v9.22523

22. Puggina CC, Amestoy SC, Fernandes HN, Carvalho LA, Báo ACP, Alves FO. Educação permanente em saúde: instrumento de transformação do trabalho de enfermeiros. Espaç Saúde [Internet]. 2015 [citado em: 18 set 2017]; 16(4):87-97. Disponível em: http://www.uel.br/revistas/uel/index.php/e spacoparasaude/article/view/22580/11

23. Miccas F, Batista NA, Batista SH. Metassíntese: uma experiência de pesquisa sobre educação permanente em saúde. In: 5 Congresso Ibero-Americano em Investigação Qualitativa [Internet]. 14-14 jul 2016. Porto, Portugal; 2016 [citado em: 18 set 2017]. p. 944-53. (Atas-Investigação Qualitativa em Saúde; vol. 2). Disponível em: http://proceedings.ciaiq.org/index.php/ciaiq 2016/article/view/841/827

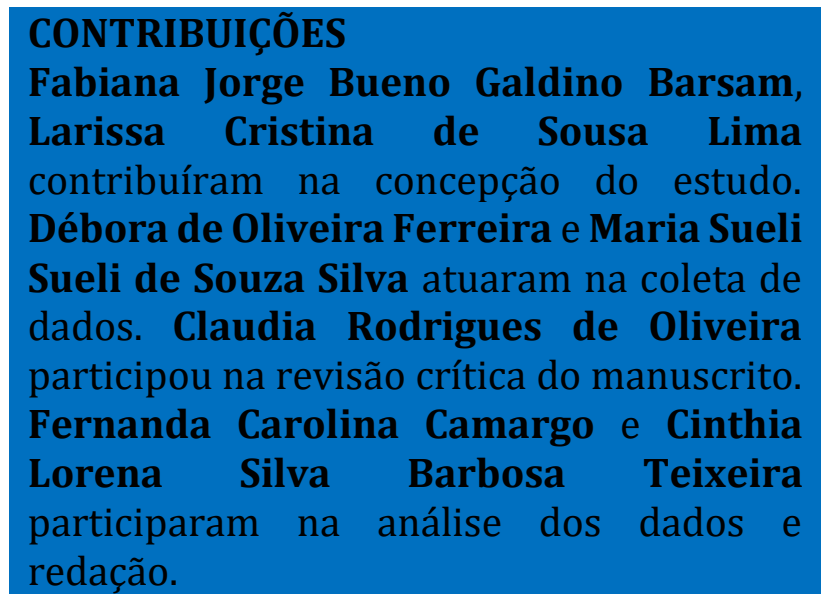




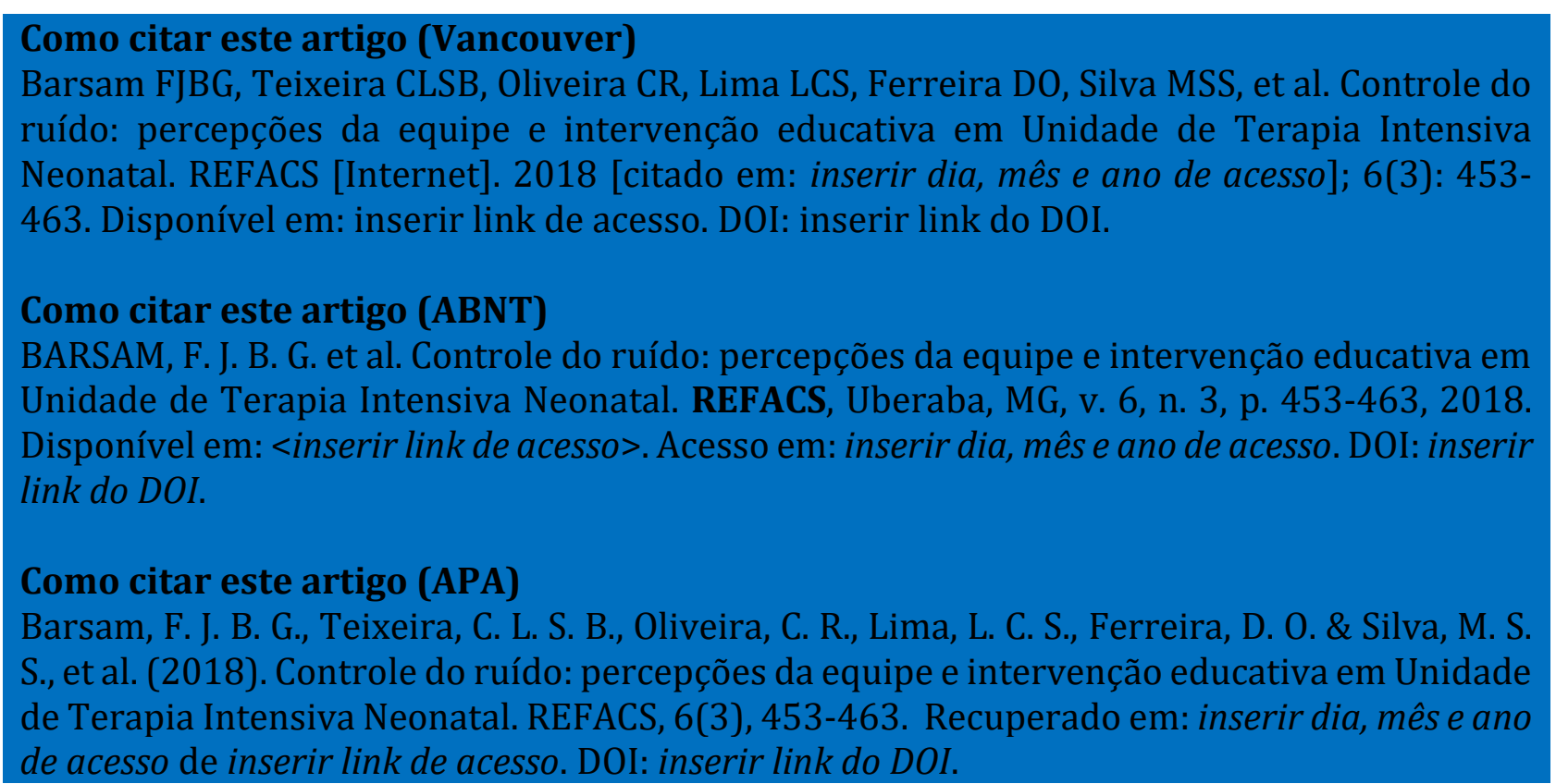

